\title{
MODELOS Y ANALOGÍAS EN LA ENSEÑNANZA DE LAS CIENCIAS NATURALES. EL CONCEPTO DE MODELO DIDÁCTICO ANALÓGICO
}

\author{
GALAGOVSKY, LYDIA y ADÚRIZ-BRAVO, AGUSTÍN \\ Centro de Formación e Investigación en Enseñanza de las Ciencias \\ Facultad de Ciencias Exactas y Naturales. Universidad de Buenos Aires \\ Ciudad Universitaria. Pabellón II. 1428 Buenos Aires. Argentina \\ E-mail: lyrgala@qo.fcen.uba.ar
}

\begin{abstract}
SUMMARY
In this paper scientific models are examined with a representational and linguistic perspective. Some didactical models are considered as raw simplifications of the original scientific models, acritically brought into the classroom. Finally, we define and exemplify the concept of didactical analogical model. Our hypothesis distinguishes between experts (teachers) and novices (students) in terms of the richness and fluency of the families of models that they use.
\end{abstract}

\section{INTRODUCCIÓN}

El concepto de modelo es uno de los pilares metateóricos sobre los que se edifican las ciencias naturales. En nuestro trabajo en la didáctica de las ciencias, nos interesa el concepto de modelo tanto desde sus aspectos lingüísticos como representacionales. En el presente artículo nos centramos en el análisis del concepto de modelo, particularizado en el pensamiento de expertos y novatos, entre los que existen algunas diferencias que se evidencian, por ejemplo, en la utilización de cualquier modelo científico en el aula. Luego, señalamos algunas características de este concepto en la epistemología contemporánea, para más tarde dedicarnos con más detalle al problema de trabajar con modelos científicos en el aula, entendiendo al profesor como experto y al alumno como novato.

Del análisis que hacemos surgen nuestras definiciones de modelo del sentido común y de modelo didáctico analógico, que comparten algunos aspectos con el modelo científico erudito y se diferencian fundamentalmente en otros aspectos relevantes que se discuten en este artículo. El análisis diferencial de estos tipos de modelos nos lleva a examinar brevemente conceptos como representación, analogía, metáfora y sus articulaciones recíprocas.

\section{CIENCIA ERUDITA Y CIENCIA ESCOLAR: LOS MODELOS MENTALES}

La comunicación entre profesorado y alumnado de ciencias naturales encuentra una serie de dificultades, una de las cuales está asociada a la brecha que se produce entre el lenguaje cotidiano (en sus aspectos sintácticos y semánticos) y el lenguaje científico erudito. Dichas 
brechas, como hemos mostrado en un trabajo anterior (Galagovsky, Bonán y Adúriz-Bravo, 1998), conducen a desencuentros y sinsentidos en la clase.

En los últimos años se está difundiendo un nuevo tipo de enseñanza de las ciencias centrado en el aprender a hablar y a escribir ciencia, es decir, centrado en la apropiación del lenguaje científico en un proceso gradual y contextualizado (Lemke, 1997; Izquierdo y Sanmartí, 1998, 1999). La problemática del contexto en el lenguaje es un importante tema de investigación, ya que ella hace evidente la fractura entre lo que llamamos ciencia erudita y ciencia escolar: el lenguaje, por ser instrumento de expresión de ambas ciencias, exhibe semejanzas y diferencias al ser usado en una u otra, y se producen deformaciones en la transición de una ciencia hacia la otra (Galagovsky et al., 1999).

Por otra parte, una dificultad importante que impide que se produzcan aprendizajes significativos son las grandes diferencias entre las diversas representaciones idiosincrásicas que construyen los alumnos acerca del mundo natural y las correspondientes representaciones científicas. Podríamos afirmar, entonces, que la diferencia entre los modelos mentales involucrados en uno y otro extremo de la comunicación entre expertos y novatos involucra tanto aspectos lingüísticos -semánticos y sintácticos- como representacionales.

En nuestro trabajo, partimos de la hipótesis de que la ciencia escolar -enseñada y aprendida en la educación obligatoria-y la ciencia erudita -también llamada de los científicos-pueden acercarse si se consideran como dos componentes articulados de una empresa social muy vasta que incide en diferentes aspectos de la vida contemporánea. Este acercamiento, que es el desvelo tanto de los diseñadores de currículo como de los docentes, ha sido encarado de muy diversas maneras. Tres grandes aproximaciones (Galagovsky et al., 1999) pueden ser:

1) Considerar que la enseñanza de las ciencias en la escuela podría seguir una secuencia lineal de contenidos conceptuales, procedimentales y actitudinales, de tal forma que se comienza con un número reducido de temáticas que se irían ampliando en cantidad a medida que avanza la escolarización. Esta aproximación sugiere que cada alumno sólo manejaría saberes científicos proporcionales a sus años de escolaridad, suponiendo el máximo conocimiento para un egresado de la titulación superior en el área.

2) Considerar que la enseñanza de las ciencias en la escuela podría consistir en una visión totalizadora de contenidos conceptuales, procedimentales y actitudinales, de tal forma que se abarcaran importantes volúmenes de información desde los primeros niveles de escolaridad, variando sus niveles de profundización conceptual a medida que ésta avanza. Esta aproximación sugiere que cada alumno tendría algún tipo de acercamiento a vastos saberes científicos, y que su dominio de la profundidad conceptual sería proporcional a sus años de escolaridad, suponiendo la máxima profundidad para un egresado de la titulación superior en el área.
3) Considerar la existencia de una ciencia escolar que involucraría una visión selectiva de contenidos conceptuales, procedimentales y actitudinales, de tal forma que la selección consistiera en un relevamiento de los conceptos estructurantes de las disciplinas científicas (Sanmartí e Izquierdo, 1997), adaptados a su máxima profundidad según las condiciones de entorno de cada situación de enseñanza-aprendizaje en particular (edad de los alumnos, recursos de diferente índole, condicionantes socioculturales, etc.). Esta aproximación sugiere que cada alumno al final de la educación obligatoria tendría un grado aceptable de alfabetización científica. Se supone que la titulación superior en un área científica aportaría gran cantidad de conocimientos específicos al quehacer profesional en dicha área, que no serían parte imprescindible del bagaje cultural de un miembro cualquiera de nuestra sociedad.

Cualquiera de estas visiones supone una fuerte continuidad entre la ciencia escolar y la erudita. Adoptar la tercera visión trae dos consecuencias fundamentales:

1) La primera consecuencia es que la ciencia escolar, la ciencia que se enseña y se aprende en la escuela, no se limita a ser una mera simplificación de la ciencia erudita, adaptada al nivel de maduración de los alumnos, sino que posee todo un arsenal de etiquetas lingüísticas, conceptos y modelos propios y originales que funcionan como facilitadores del acceso del alumnado a las formas más altas de representación científica.

A partir de las investigaciones en psicología y en ciencia cognitiva, sabemos que apropiarse de cualquier aspecto de la realidad supone representárselo, es decir, construir un modelo mental de esa realidad (Izquierdo, 1999). Este modelo mental está constituido fundamentalmente por aspectos lingüísticos y representacionales. Analogando el concepto de modelo mental al de modelo científico, podríamos decir que aprender ciencia implica manejar el lenguaje y las representaciones de la ciencia erudita.

Hablar con el lenguaje y las representaciones propias de la ciencia escolar sería un paso necesario en el camino hacia aprender la ciencia de los científicos. Pretender que desde un primer momento los alumnos utilicen un lenguaje estrictamente científico no necesariamente es una manifestación de que la información que manejan está sustentada en representaciones científicas cercanas a las propuestas por la ciencia erudita en ese campo. En efecto, muchas veces se ha verificado la declamación de textos aprendidos memorísticamente pero, luego de un tiempo, aprendizajes correctos aparentemente consolidados se borran, apareciendo nuevamente ideas erróneas, no correspondientes al modelo científico apropiado, sino respondiendo a lo que llamaremos modelos del sentido común o del pensamiento espontáneo, que han sido ampliamente estudiados (Gutiérrez, 1999).

2) La segunda consecuencia es que el otorgar autonomía y carácter propio a la ciencia escolar abre la posibilidad de que ésta sea una entidad independiente, en evolución, que crea sus propias representaciones, herramientas y lenguaje, adecuándolos al objetivo de permitir la transi- 
ción hacia la ciencia erudita. Esta nueva forma de entender la enseñanza de las ciencias en la escuela implica cambios, aún no completamente estudiados, que se organizan alrededor de lo que muchos autores han dado en llamar un enfoque constructivista (Porlán, 1998). En el ámbito de la didáctica de las ciencias, este tema se aborda como un fenómeno muy complejo de interacción entre dos formas de ciencia, entre las cuales existen tanto continuidades como rupturas.

Centrándonos específicamente en los modelos mentales como unidad de análisis, podemos encontrar notables diferencias entre el lenguaje de la ciencia escolar en los niveles iniciales, que es muy cercano al del sentido común, y el lenguaje más complejo de la ciencia erudita contemporánea. A lo largo de la educación obligatoria y superior es plausible suponer una serie de transiciones graduales entre uno y otro lenguaje, auxiliadas por diversas estrategias linguísticas. Lo mismo vale para las representaciones internas, que gobiernan grandemente el uso del lenguaje en ciencias. Podemos afirmar que, cuando se es capaz de hablar el lenguaje de la ciencia, las representaciones internas del mundo se corresponden con aquéllas que da la ciencia erudita (Lemke, 1997).

\section{Los modelos del sentido común y los de la ciencia erudita}

Los modelos del sentido común se construyen idiosincrásicamente a partir de la experiencia cotidiana en el mundo natural y de las interacciones sociales; son eminentemente figurativos, casi pictóricos. El sentido común supone una base de realismo ingenuo, por la cual el modelo funciona casi como un calco de la realidad tal y como ésta es captada por los sentidos, y entonces no requiere de entidades instrumentales auxiliares. Para cada porción de la realidad que es objeto de un problema, se formula en general un único modelo rígido. El modelo funciona como una representación de primer orden, analogada de la realidad, a la que a menudo sustituye. Este modelo se pone en acción con una serie de reglas lógicas que frecuentemente difieren de las del pensamiento hipotético-deductivo riguroso. Se trata a menudo de una serie de causaciones lineales e irreversibles.

Los modelos científicos se construyen mediante la acción conjunta de una comunidad científica, que tiene a disposición de sus miembros herramientas poderosas para representar aspectos de la realidad. Inicialmente, la ciencia procede a un recorte de la realidad que se considera teóricamente relevante. Este recorte abstrae, simplifica, reestructura y analoga los diferentes elementos, dando lugar a un sistema en particular. Este sistema, a su vez, es sólo uno de los posibles sistemas que esa porción de realidad seleccionada admite.

Los modelos científicos pasan así a ser representaciones de segundo orden, hechas sobre los sistemas, que ya son en sí mismos abstracciones de la realidad. Así, los modelos resultan representaciones sumamente abstractas, escasamente figurativas, más cercanas a una posición abiertamente instrumental que al realismo ingenuo del sentido común (Giere, 1999). En esta postura, los modelos son considerados herramientas de representación teórica del mundo, auxiliares para explicarlo, predecirlo y transformarlo (Adúriz-Bravo, 1999).

Por otra parte, los modelos ganan en movilidad y se estructuran en familias, con distintos niveles de complejidad de acuerdo con el tipo de problemas para los cuales se utilizan. Estos modelos coexisten en la ciencia, y el científico se sirve de ellos según las circunstancias particulares en las que se encuentra. Para la misma realidad a explicar tenemos, entonces, varias familias de modelos adecuadas a cada problema científico específico y al enfoque con que éste es tratado (Giere, 1992, 1999).

Otro fenómeno interesante es que la constitución de los modelos científicos supone la utilización de entidades instrumentales auxiliares, que aportan datos más allá de los captados por los sentidos con ayuda de los instrumentos tecnológicos. Asimismo, la comunicación de modelos científicos entre expertos utiliza también elementos del lenguaje literario que enriquecen la descripción del modelo científico, como son la analogía y la metáfora.

Las analogías han jugado un papel muy importante en el desarrollo histórico del conocimiento científico (Hesse, 1966). Un ejemplo muy conocido es la analogía del «budín con pasas» para el modelo atómico de Thomson (premio Nobel de 1902). Estas formas expresivas permiten representaciones más significativas del contenido del modelo y transferencias de éste a otros campos. Así, el lenguaje científico se enriquece con una serie de estrategias que podrían haberse supuesto exclusivas del lenguaje literario, pero que juegan un importante papel en la construcción y consenso de significaciones en la ciencia (Gross, 1990).

Esta imagen de un bagaje lingüístico que se va enriqueciendo y complejizando a lo largo de la educación nos permite centrar nuestra atención en el estudio del desempeño lingüístico de expertos y novatos en ciencias naturales. El análisis de tal desempeño, a su vez, nos posibilita cierto acceso a las representaciones mentales que unos y otros ponen en juego frente a la realidad natural.

\section{APROXIMACIÓN AL CONCEPTO DE MO- DELO}

\section{Los modelos en la epistemología}

En la epistemología, la noción de modelo científico ha estado desde siempre estrechamente ligada a la de teoría. Sin embargo, en los últimos años asistimos a un cambio importante en la visión disciplinar de las relaciones entre una y otra entidad. Se están difundiendo una serie de investigaciones y teorizaciones específicas alrededor de los modelos con cierta independencia de los tradicionales intentos de formalización de las ciencias en grupos de teorías (Giere, 1992, 1999). 
En efecto, el concepto de modelo está recibiendo una mayor atención en la epistemología, a raíz, entre otras cosas, de las investigaciones específicas en psicología del aprendizaje, ciencia cognitiva y didáctica de las ciencias, que lo han señalado como un concepto poderoso para entender la dinámica de la representación que tanto científicos como estudiantes se hacen del mundo (Izquierdo, 1999).

Desde esta visión menos axiomática, las ciencias naturales, en tanto que vastos conjuntos de modelos sobre el mundo, contienen en sí mismas formalización e interpretación en diversa medida. Es decir, combinan elementos sintácticos, que son los que hacen al lenguaje en el cual se expresan sus modelos, y elementos semánticos, que hacen a los contenidos del mundo asociados a los mismos.

Las entidades lingüísticas con las que trabaja la ciencia son verdaderos operadores en los modelos, que pretenden ser la representación teórica de la realidad. Esos modelos articulan el conjunto de representaciones asociadas a la explicación científica. Así, los modelos científicos son las mediaciones entre el sistema formal «teórico» y su interpretación «empírica» (Adúriz-Bravo, 1999).

\section{Algunas características de los modelos científicos para ser tenidas en cuenta en el aula}

Algunas de las características más importantes de los modelos científicos son poco explicitadas durante el trabajo en el aula; sin embargo, se trata de rasgos esenciales porque denotan una posición epistemológica frente al conocimiento científico enseñado. Entre ellas, podemos mencionar tres:

1) Los modelos como construcciones provisorias y perfectibles. A lo largo de la historia de la ciencia, los modelos se han ido sucediendo en el avance hacia formas cada vez más poderosas, abarcativas y útiles de explicar la realidad. La consecuencia más importante de esta visión de la historia de la ciencia es la de que todo modelo, como tal, es provisorio y perfectible, y que ningún modelo científico posee la verdad absoluta y definitiva sobre nada.

2) Los modelos científicos alternativos pueden no ser compatibles entre sí. Dos modelos que pretenden explicar simultáneamente la misma porción de la realidad no son necesariamente incompatibles; pero la incompatibilidad aparece si ellos no comparten sus presupuestos de partida, es decir, se inscriben en diferentes escuelas teóricas o paradigmas. Tal situación de competencia se ha dado muchas veces a lo largo de la historia de la ciencia, y el proceder científico generalmente elige el modelo que usará en base a su sencillez, su riqueza teórica y su poder explicativo, teniendo los datos experimentales una importancia menor en esta elección. Por ejemplo, el modelo heliocéntrico del universo de Copérnico era mucho más sencillo que el modelo geocéntrico de Ptolomeo, aunque no necesariamente explicaba, en el momento de su formulación, muchas más observaciones que este último.

Surge de aquí que, en el aula, todos los modelos, como teorizaciones personales, deberían ser respetados inicialmente en pie de igualdad, y que, si se elige uno, es por consenso (no por imposición) y en base a su utilidad teórica para la explicación. Dicha elección, entonces, está guiada por el modelo científico que constituye el contenido mismo de enseñanza. Esta guía desde el profesorado permite evitar el relativismo subyacente a muchas propuestas didácticas constructivistas, que dan igual estatuto a cualquier explicación sobre el mundo (Izquierdo, 1999).

3) Los modelos alternativos no siempre son sucesivamente incompatibles entre sí. Un modelo que reemplaza a otro no suele contener al anterior, puesto que implica una nueva forma de pensar y modelar la realidad en distintos términos: por ejemplo, la teoría de la relatividad de Einstein reemplaza a la teoría clásica de Newton destruyendo sus nociones de tiempo y espacio. Lo dicho no quita que un nuevo modelo sí contiene gran parte de las explicaciones y predicciones del anterior y añade nuevas, por lo que sustituye al otro en la ciencia. Sin embargo, el reemplazo de un modelo por otro no comporta el abandono definitivo del primero. Instrumentalmente, pueden utilizarse modelos perimidos cuando facilitan la manipulación formal y constituyen aproximaciones sencillas y legítimas a un problema científico; este procedimiento es usual en la investigación tecnológica.

De esto se sigue que todos los temas son tratables por aproximaciones sucesivas (un abordaje «en espiral») y que los contenidos que se estudian sirven de base provisoria para nuevas formas de pensarlos en el futuro; nunca quedan cerrados definitivamente. Además, algunos modelos históricamente propuestos siguen formando parte activa de la ciencia actual (esta consideración es particularmente importante en el contexto de educación).

\section{LOS MODELOS CIENTÍFICOS EN EL AULA: UN PROBLEMA DIDÁCTICO A RESOLVER}

\section{¿Es el modelo didáctico una «re-representación» del modelo científico?}

Un modelo científico contiene la articulación de un gran número de hipótesis de altísimo nivel de abstracción atinentes a un cierto campo problemático de la realidad. El alto grado de formalización de un tal modelo hace que esté a menudo fuera de las capacidades operatorias y de la disponibilidad de conocimientos previos de los alumnos de la escuela primaria y secundaria.

Aprender ciencias naturales en la escuela requeriría, entonces, reconstruir los contenidos científicos por medio de una imagen didáctica adecuada que los «lleve al aula». Sin embargo, lo que suele ocurrir es que se utilizan modelos científicos simplificados, que tienen 


\section{INVESTIGACIÓN DIDÁCTICA}

significado para el nivel de erudición del profesor, pero que no encuentran referente en la estructura cognitiva de los alumnos. En estas circunstancias, los alumnos deben incorporar memorísticamente un modelo que no es completamente científico y que, además, les resulta escasamente significativo.

Revisando los textos escolares (Adúriz-Bravo y Galagovsky, 1997) encontramos algunas cuestiones en la utilización de los modelos científicos que podrían propiciar confusión en los alumnos. La utilización indiscriminada, secuencial y alternativa de diferentes modelos científicos, en sus representaciones más simplificadas, carentes de contexto histórico y, por lo tanto, sin indicación de sus alcances y limitaciones, mezclada con herramientas simbólicas que han surgido de convenciones y acuerdos entre científicos, pero que se enseñan como normativas, nos conducen a sugerir que algunos modelos didácticos utilizados resultan de combinar, sin jerarquía y desordenadamente, modelos, instrumentos, representaciones y recursos sintácticos y semánticos provenientes de la ciencia erudita.

Así, los modelos didácticos alternativos sobre el mismo tema aparecen como aproblemáticamente compatibles en un mismo curso, libro o ciclo escolar; además son entendidos como sucesivamente compatibles sin que aparezca ningún conflicto entre ellos. En ningún momento se reflexiona sobre la validez contextual de cada una de las representraciones y sobre las posibles incongruencias entre unas y otras. Mucho menos se intenta vincular estas familias de representaciones con las que traen construidas los alumnos.

La tabla I resumiría las diferencias más notorias entre los modelos de la ciencia erudita y estos «modelos didácticos».

Estos comentarios nos llevan a pensar que, si los mensajes del discurso escolar y de las fuentes de información alternativas son poco significativos, los alumnos tenderán a otorgarles -con cierto esfuerzo cognitivo- la significación más conveniente según su sentido común personal e idiosincrásico, es decir, acomodarlos a sus propios modelos previos. Es así como muchos aprendizajes podrán ser erróneos desde el punto de vista científico, y constituirse posteriormente en obstáculos epistemológicos y resistencias para acceder al conocimiento científicamente validado.

Tabla I

Algunas diferencias entre los modelos científicos y los «modelos didácticos» simplificados presentes a menudo en los libros de texto.

\begin{tabular}{|c|c|}
\hline Modelo de la ciencia erudita & Modelo didáctico \\
\hline $\begin{array}{l}\text { - Es una construcción provisoria, altamente } \\
\text { convencional, perfectible y contextualizada } \\
\text { históricamente. }\end{array}$ & $\begin{array}{l}\text { - En general, proviene de un modelo científico perimido } \\
\text { y simplificado, descontextualizado de cualquier momento } \\
\text { histórico, con la consiguiente apariencia de «verdad } \\
\text { imperecedera». }\end{array}$ \\
\hline $\begin{array}{l}\text { - Dos modelos científicos alternativos sobre } \\
\text { el mismo tema pueden ser simultáneamente } \\
\text { incompatibles si se han propuesto desde } \\
\text { diferentes escuelas teóricas. }\end{array}$ & $\begin{array}{l}\text { - Los modelos didácticos alternativos sobre el mismo tema } \\
\text { parecen siempre simultáneamente compatibles en un mismo } \\
\text { curso, libro o ciclo escolar. }\end{array}$ \\
\hline $\begin{array}{l}\text { - Dos modelos científicos alternativos sobre } \\
\text { el mismo tema pueden ser sucesivamente } \\
\text { incompatibles si hay un cambio en los conceptos } \\
\text { teóricos involucrados. }\end{array}$ & $\begin{array}{l}\text { - Los modelos didácticos alternativos sobre el mismo tema } \\
\text { parecen siempre sucesivamente compatibles; no se habla } \\
\text { de su evolución histórica ni conceptual. }\end{array}$ \\
\hline $\begin{array}{l}\text { - Surgen del consenso de científicos que discuten } \\
\text { en comunidad. }\end{array}$ & $\begin{array}{l}\text { - Se imponen desde el texto o en el discurso escolar, } \\
\text { no se explicita su naturaleza convencional. }\end{array}$ \\
\hline $\begin{array}{l}\text { - Se utilizan para explicar los fenómenos } \\
\text { que la experimentación de un determinado } \\
\text { momento histórico evidencia. }\end{array}$ & $\begin{array}{l}\text { - Se enseñan como verdades intrínsecas, no para explicar } \\
\text { fenómenos; se considera necesario enseñar «el último modelo», } \\
\text { por lo tanto, no hay contradicciones entre hechos experimentales } \\
\text { y modelos anteriores. }\end{array}$ \\
\hline
\end{tabular}




\section{LAS REPRESENTACIONES DIDÁCTICAS}

En relación con lo expresado en el punto anterior, cabe denunciar la ambigüedad con que utilizamos el término de modelo didáctico. Proponemos, desde este análisis, considerar una clasificación de los recursos didácticos que pueden desplegarse en las clases de ciencias naturales. Hablaremos de representaciones científicas, representaciones concretas, análogos concretos y modelos didácticos analógicos.

\section{Representaciones científicas}

Son imágenes visuales obtenidas por alguna mediación instrumental más o menos sofisticada, tales como imágenes digitalizadas, espectros de cualquier tipo, micrografías, rayos X. El referente de este tipo de representación es un concepto científico, visualizado mediante artificios tecnológicos. Ejemplos de esta categoría serían: una imagen de microscopía electrónica, un electrocardiograma, la fotografía infrarroja de una superficie, una ecografía, una resonancia magnética nuclear, un espectro de masas, una foto satelital.

Un experto puede decodificar fácilmente este tipo de representaciones, interpretar sus alcances, aplicaciones y limitaciones, sus escalas de trabajo, la sensibilidad del método instrumental utilizado, el grado de distancia entre la reconstrucción visual del concepto científico propiamente dicho y su representación científica. Un novato, en cambio, suele aceptar este tipo de representación como «verdadera», es decir, realizar una interpretación icónica estrecha de la misma, sin comprender la naturaleza mediacional de los instrumentos utilizados.

\section{Representaciones concretas}

Son representaciones visuales de ciertas imágenes asociadas a algún modelo científico en particular; pueden ser dibujos, proyecciones bidimensionales u objetos tridimensionales. El referente de este tipo de representación es también un concepto científico, reconstruido mediante artificios pictóricos, generalmente simplificadores del concepto más complejo. Ejemplos de este tipo serían: el dibujo de un orbital, el esquema de una célula, un modelo molecular (de bolitas y palillos), una maqueta del sistema solar, una simulación obtenida mediante ordenadores u hologramas.

Un experto comprende que este tipo de representación involucra una simplificación del concepto científico referente; interpreta sus alcances, aplicaciones y limitaciones, sus escalas de trabajo y el grado de distancia entre el concepto científico propiamente dicho y su representación concreta. El novato, en cambio, suele aceptar este tipo de representación como «verdadera», sustituyendo a la entidad científica, sin comprender la naturaleza mediática y metafórica de las convenciones, correspondencias y simplificaciones utilizadas.

\section{Análogos concretos}

Son dispositivos didácticos facilitadores del aprendizaje de conceptos abstractos (Glynn, 1990), los cuales utilizan conceptos y situaciones que tienen un claro referente en la estructura cognitiva de los alumnos; este referente se relaciona analógicamente con los conceptos científicos cuyo aprendizaje se quiere facilitar (Galagovsky, 1993a). Ejemplos de análogo concreto serían utilizar un resorte para representar los movimientos cuantificados de las uniones interatómicas en una molécula-verificables a través de un espectro infrarrojo-; o el uso de un sistema hidráulico para representar un circuito eléctrico simple con elementos en paralelo y en serie.

Al operar una transposición sobre los saberes eruditos para transformarlos en contenidos escolares, puede fabricarse sobre los contenidos y procedimientos científicos una nueva representación analógica mediada por conceptos cotidianos o ficticios cercanos al conocimiento del sentido común de los alumnos. Nuestra adjetivación de concreto para el concepto de análogo hace hincapié en que la intención, al crear una analogía, es apelar a conceptos de significación ya conocida por los alumnos. Suponemos, entonces, que estos novatos podrán operar sobre dichos contenidos desde su pensamiento operatorio concreto-tomando este término de la teoría piagetiana (Piaget e Inhelder, 1959)-, y estimamos que, mediante estrategias didácticas apropiadas, ellos podrán también desarrollar un pensamiento operatorio formal hipotético-deductivo sobre dichos contenidos analógicos.

Por otra parte, desde la perspectiva constructivista cabe considerar que el razonamiento analógico es la llave que permitiría el acceso a los procesos de aprendizaje, ya que todo nuevo conocimiento incluiría una búsqueda de aspectos similares entre lo que ya se conoce y lo nuevo, lo familiar y lo no familiar (Pittman, 1999). El uso de analogías puede jugar, entonces, un papel muy importante en la reestructuración del marco conceptual de los alumnos novatos, puede facilitar la comprensión y visualización de conceptos abstractos, puede despertar el interés por un tema nuevo, y puede estimular al profesorexperto a tener en cuenta el conocimiento previo de los alumnos.

Generalmente, son los profesores los que generan la analogía porque conocen el tema desde la perspectiva científica y, al simplificarlo, pretenden transmitir sus propiedades relevantes a objetos o situaciones cercanas al sentido común. El experto releva qué aspectos puede o quiere simplificar del concepto científico referente; interpreta sus alcances, aplicaciones y limitaciones, y el grado de distancia entre el concepto científico propiamente dicho y su analogía. Pocos, pero interesantes, son los casos en los que se permite que los alumnos novatos generen analogías a partir de haber estudiado un tópico científico. El novato pasivo en la recepción de una analogía puede llegar a percibirla como una información facilitadora de la comprensión del tema pero, a menudo, reclama que es más información a estudiar, porque la analogía es complicada o porque no ha comprendido las 
similitudes que guarda con el modelo científico; otras veces, sólo ha comprendido la analogía inicial pero no el contenido científico meta (Pittman, 1999).

\section{Modelo didáctico analógico}

Para marcar un avance respecto del concepto de análogo concreto -como un ejemplo que da el profesor y que apela a componentes del sentido común o de la vida cotidiana de los alumnos- y, simultáneamente, para acercarnos al concepto de modelo científico -en el sentido en que se usa este concepto en la ciencia erudita para justificar, interpretar y predecir fenómenos-, definimos el modelo didáctico analógico (MDA) como dispositivo de la ciencia escolar.

La idea básica para construir un modelo didáctico analógico es conocer profundamente el tema que se quiere enseñar, abstraer sus conceptos nucleares y las relaciones funcionales entre dichos conceptos y traducir todo a una situación, lo más inteligible posible para el alumnado, proveniente de la vida cotidiana, de la ciencia ficción o del sentido común. Hasta aquí podrían no verse claramente las diferencias con un análogo concreto, al cual ciertamente se parece; sin embargo, la distinción fundamental radica en el contexto didáctico con el que se lo trabaja en la clase. La estrategia didáctica para operar con un MDA requeriría tres momentos diferenciados:

1) En primer lugar, el MDA se aborda generalmente antes de que el tema específico -su referente científicohaya sido tratado. Los alumnos, comprendiendo esta situación analógica inicial, pueden formular hipótesis sobre qué, por qué, cómo y cuándo ocurren diferentes fenómenos en el análogo, que luego podrán relacionarse con los contenidos, procedimientos y lenguaje de la ciencia erudita. Dentro del MDA se establecen hipótesis, se argumenta, se justifican fenómenos, se predicen situaciones que pueden, incluso, ponerse a prueba.

Un registro escrito de esta primera etapa es fundamental para facilitar el momento de la metacognición, del que se habla más abajo, en el que se analizan las ventajas de haber trabajado en los planos de la ciencia escolar y la ciencia erudita mediante la toma de conciencia sobre los alcances y limitaciones del análogo trabajado. Este registro escrito puede ser una tabla, un mapa o una red conceptual (Galagovsky, 1996), un esquema, un relato, la contestación a un cuestionario, etc.

2) En segundo lugar, una vez trabajada la situación analógica desde el conocimiento propio de los alumnos, se presenta la información proveniente de la ciencia erudita. Esta información puede tener el formato de un texto o de una exposición del profesor, y está descrita con el lenguaje más apropiado de la ciencia erudita adaptado para ese dado nivel de escolaridad. Es decir, primero se trabaja con el MDA, desde un contexto de ciencia escolar, con un lenguaje más cercano al cotidiano, y sólo después se entrega la información más propia de la ciencia erudita. El trabajo de los alumnos consis- tirá, entonces, en la elaboración de nuevas hipótesis que relacionen el MDA y el modelo científico a través de sus similitudes y diferencias. Nuevamente, la consigna didáctica debería ser precisa y estar apoyada en un cuestionario o en la confección de una tabla, un mapa o una red conceptual, que puedan compararse luego con aquellos escritos que resultaron de la primera etapa sobre el MDA.

3) Finalmente, el momento de la metacognición, entendida como autogestión del aprendizaje (Monereo Font, 1995) o como una toma de conciencia del novato sobre el salto cognitivo que se ha logrado en el tema (Galagovsky, 1993a), constituye el propósito didáctico de la última etapa del MDA. En este momento se requiere por parte del alumnado un análisis riguroso para explicitar las transposiciones que operaron en los procesos de analogación: los recortes, simplificaciones y aproximaciones que se produjeron, las transferencias y desplazamientos del contenido, los rangos de validez conceptual y operacional, y el conjunto de operaciones inversas que nos permiten recuperar el modelo original (la condición de reversibilidad). Esta etapa de metacognición, en tanto que supone un tipo de pensamiento del más alto nivel de conceptualización y la revisión de los mecanismos propios de adquisición del conocimiento, es la etapa de mayor resistencia por parte de los alumnos novatos, y tanto mayor es la dificultad cuanto menor es la edad de los alumnos o su grado de ejercitación en este tipo de pensamiento.

\section{UN EJEMPLO DE MODELO DIDÁCTICO ANALOGICO (MDA)}

Se trata de una experiencia realizada para la enseñanza de la estructura de la célula. Los alumnos pertenecen a una primera asignatura de biología del ciclo universitario (edad de 18 años como mínimo); el estudiantado tiene grandes desniveles en cuanto a los conocimientos previos que trae al curso. El objetivo de la clase es comprender un modelo representativo de la dinámica celular a través de interpretar la naturaleza de la membrana citoplasmática, barrera que sirve como límite y, a su vez, como conexión con el medio externo, involucrando relaciones entre la organización bioquímica, anatómica y fisiológica de la unidad vital de un organismo vivo. Para ello se tiende una analogía con las diversas entradas a una casa (Apéndice).

En las clases anteriores se han enseñado los componentes bioquímicos de las membranas celulares. Estos temas previos habían sido fundamentalmente descriptivos y la experiencia indica que los alumnos tienen dificultades para comprender las relaciones entre dichas moléculas y sus respectivas funciones en cuanto a la dinámica de los procesos celulares. Estas dificultades fuerzan a una compartimentación memorística en la forma de aprender, con la consiguiente falta de integración de significados sobre las relaciones biológicas de estructura y función. 


\section{Etapas, momentos, actividades y registros en el MDA de la membrana celular}

La primera etapa didáctica de este MDA tiene tres momentos:

1) En un primer momento, la clase se divide en grupos de 4-5 estudiantes, con la consigna de resolver por escrito, en forma muy breve y en unos diez minutos, el cuadro I que se muestra en el apéndice (Galagovsky, 1993b).

2) A continuación, cada grupo expone su producción. Esta instancia de explicaciones a partir de los conocimientos de los alumnos es fundamental, pues allí surgen espontánea y explícitamente sus ideas previas, ya fueran aprendidas o intuitivas, a veces asociadas a una falta de vocabulario preciso y con cierta desconexión entre las palabras utilizadas y la significación que se da a las mismas. Se hacen evidentes, además, las significaciones idiosincrásicas tanto relativas a la interpretación de los fenómenos como a la explicación de los mismos y al vocabulario utilizado.

En este segundo momento, el docente debe ayudar al alumnado a tomar conciencia sobre estas disyuntivas; es el momento en el que se instala el llamado conflicto cognitivo. Es en esta puesta en común donde se socializa el conflicto cognitivo -necesariamente individual en un comienzo-. Durante el debate, el objetivo es arribar a un consenso en las representaciones mentales y en el vocabulario utilizado para describir dichas representaciones. Ese vocabulario puede provenir tanto de las ciencias como del léxico cotidiano; por lo tanto, será necesario compatibilizar las significaciones dadas a las palabras más allá de la discusión de casa caso particular del cuadro I. Por ejemplo, no tiene sentido discutir con detalles el fenómeno físico que explica cómo se desplaza el humo, sino relevar las diferentes interpretaciones provenientes de los alumnos, ayudarlos a eliminar variables superpuestas (p.e., cuestionar sobre por qué el humo se desplaza de un lugar donde está más concentrado a otro lugar), ayudarlos a despejar malas interpretaciones (p.e., cuestionarlos sobre qué es el viento, para el caso en que contesten que el desplazamiento del aire se debe al viento), y centrarse en llegar a un acuerdo sobre la forma de nombrar un dado fenómeno que hace referencia a un aspecto general del desplazamiento de cada tipo de agente.

3) Aquí tiene lugar el tercer momento de esta etapa del MDA: con la coordinación del docente debe arribarse a una suerte de consenso mínimo tanto sobre la naturaleza de cada uno de los fenómenos del cuadro como del vocabulario científico más apropiado para describirlos. En el cuadro II del apéndice se muestran ejemplos de las respuestas elaboradas por los alumnos. En la columna derecha del cuadro II se muestra una síntesis del acuerdo al cual debería llegarse al finalizar la puesta en común.

Una vez consensuada la terminología y su asociación a los fenómenos del cuadro II (columna de la derecha), comienza la segunda etapa didáctica del MDA. En esta etapa, cada grupo tiene una serie de artículos de divulga- ción científica o partes de textos que deben ser leídos con la consigna de asignar una forma de transporte a cada tex to y asociarla con alguno de los fenómenos discutidos en el análogo.

Algunos ejemplos de las asociaciones posibles son:

a) el caso del humo como el agua intracelular y extracelular;

b) el caso de las moscas y mosquitos como transporte activo;

c) el caso del cobrador de impuestos como el de las hormonas;

d) el caso del elefante como macromoléculas no absorbibles;

e) el caso del piano como ejemplo del fenómeno de fagocitosis;

f) el caso del ladrón como ejemplo de venenos y virus; g) el caso de la carta como el transporte de sodio y potasio.

Finalmente, la tercera etapa del MDA requiere favorecer procesos metacognitivos, que surgirán de cuestionar el propio proceso realizado: ¿Qué es lo que aprendimos y cómo lo aprendimos? ¿Cuáles son los alcances y limitaciones de las asociaciones que se hicieron entre los casos científicos de los artículos y sus simplificaciones asociadas a los fenómenos del cuadro? ¿Cuáles son las relaciones entre estructura y función en una casa y en una célula?

Generalmente, esta etapa requiere unos minutos de reflexión individual, luego de pequeño grupo y, finalmente, una puesta en común, donde el docente ayuda a la toma de conciencia no sólo sobre el tema científico específico, sino también sobre las estrategias cognitivas de apropiación del conocimiento, basadas en las analogías.

\section{CONCLUSIONES}

En las secciones anteriores expusimos las herramientas teóricas que nos fueron necesarias para formular un primer modelo del proceso de modelización en la enseñanza de ciencias naturales. Suponemos que los profesores, en tanto que expertos, y los alumnos, en tanto que novatos, poseen un arsenal muy diferente de modelos y los utilizan en forma bien distinta. Los expertos poseen una red muy compleja y rica de representaciones ubicadas en numerosos órdenes y conectadas entre sí por correspondencias, analogías, semejanzas y metáforas. Son capaces de moverse con soltura en esta red, cambiando de modelo según las necesidades de su propia actividad. Y, lo que es fundamental, conocen para cada modelo el conjunto de hipótesis teóricas que conectan 
dicho modelo con la realidad que se pretende modelizar. Esto es lo que Giere (1999) ha dado en llamar realismo perspectivo.

Los expertos utilizan representaciones explícitas e implícitas, con una movilidad entre las mismas (saltos de una a otra) que los alumnos pueden no percibir fácilmente. Además, y con fines didácticos, se utilizan simplificaciones de modelos complejos, que se alejan de éstos, siendo esta diferencia clara para el experto y no evidente para el que aprende. Como resultado de estas imágenes superpuestas o representaciones alternativas no explícitas, los alumnos construyen su propio modelo de representación del fenómeno en cuestión. Esta movilidad mental del experto que salta de un tipo de representación a otra en su enseñanza, con cabal conocimiento de lo que hace y por qué lo hace, choca con la rigidez representacional del alumno, que, a falta de vocabulario específico y de conceptos que le permitan anclar en su estructura cognitiva el nuevo conocimiento, debe generalmente renunciar a dar al fenómeno la significación que podría darle el científico, ensayando en su lugar una modalidad de aprendizaje memorístico y compartimentado, y ocultando sus propias ideas sobre la situación.

La utilización de diferentes tipos de representación del conocimiento tiende a favorecer la visualización

\section{REFERENCIAS BIBLIOGRÁFICAS}

ADÚRIZ-BRAVO, A. (1999). «Elementos de teoría y de campo para la construcción de un análisis epistemológico de la didáctica de las ciencias». Tesis de maestría. Universitat Autònoma de Barcelona.

ADÚRIZ-BRAVO, A. y GALAGOVSKY, L. (1997). Modelos científicos y modelos didácticos en la enseñanza de las ciencias naturales. Actas de la X Reunión de Educación en Física. Mar del Plata. Argentina.

GALAGOVSKY, L.R. (1993a). Hacia un nuevo rol docente. Una propuesta diferente para el trabajo en el aula. Buenos Aires: Troquel.

GALAGOVSKY, L.R. (1993b). Hacia un mejor aprendizaje. Claves en psicoanálisis y medicina, 4, pp. 13-16.

GALAGOVSKY,L.R.(1996). Redes conceptuales: aprendizaje, comunicación y memoria. Buenos Aires: Lugar Editorial. de conceptos abstractos. Estas representaciones, a menudo analógicas, pueden ser utilizadas de diversas formas en la clase, con variados niveles de efectividad en el aprendizaje. Nuestra propuesta de clasificación de las representaciones, y las recomendaciones sobre algunos cuidados a tener en cuenta con las representaciones analógicas, pretende ayudar a que la tendencia natural de los alumnos a dar significación a los contenidos nuevos por asociación con otros conocimientos significativos ya disponibles no quede librada a estrategias sólo controladas por su sentido común. Es de suponer que en el intercambio con el grupo de pares y con el experto se forjan significaciones más precisas y más cercanas a las de la ciencia erudita.

Nuestra concepción de la didáctica de las ciencias naturales, en relación con el desarrollo de una ciencia escolar que elabora sus propios modelos para enseñar en función de ayudar al novato a percibir y apropiar las formas de representación, los modelos, las estrategias cognitivas y el vocabulario específico de los expertos, apunta al diseño de interacciones sociales que faciliten tanto el alcance de nuevos aprendizajes significativos como la toma de conciencia sobre lo aprendido y las estrategias cognitivas empleadas (Vygostsky, 1993).
GALAGOVSKY, L.R., BONÁN, L. y ADÚRIZ-BRAVO, A. (1998). Problemas con el lenguaje científico en la escuela. Un análisis desde la observación de clases de ciencias naturales. Enseñanza de las Ciencias, 16(2), pp. 315-321.

GALAGOVSKY, L.R., MORALES, L., BONÁN, L., ADÚRIZBRAVO, A. y MEINARDI, E. (1999). El modelo de ciencia escolar: una propuesta de la didáctica de las ciencias naturales para articular la normativa y la realidad del aula. Actas de la XI Reunión de Educación en Física. Mendoza. Argentina.

GIERE, R. (1992). La explicación de la ciencia. México: Consejo Nacional de Ciencia y Tecnología. (Ed. orig. en inglés de 1988)

GIERE, R. (1999). Del realismo constructivo al realismo perspectivo. Enseñanza de las Ciencias, núm. extra, pp. 9-13. 
GLYNN, S.M. (1990). La enseñanza por medio de modelos analógicos, en Denise, K. (comp.). El texto expositivo. Buenos Aires: Aique.

GROSS, A. (1990). The rhetoric of science. Cambridge: Harvard University Press.

GUTIÉRREZ, R. (1999). La causalidad en los razonamientos espontáneos. Enseñanza de las Ciencias, núm. extra, pp. 31-61.

HESSE, M. (1966). Models and analogies in science. Notre Dame: University of Notre Dame Press.

IZQUIERDO, M. (ed.) (1999). Aportación de un modelo cognitivo de ciencia a la enseñanza de las ciencias. Enseñanza de las Ciencias, núm. extra.

IZQUIERDO, M. y SANMARTÍ, N. (1998). Ensenyar a llegir $i$ a escriure textos de ciències de la naturalesa, en Jorba, J., Gómez, I. y Prat, À. (comps.). Parlari escriure per aprendre. Ús de la llengua en situació d'ensenyament-aprenentatge de les àrees curriculars. Bellaterra: UAB-ICE.

IZQUIERDO, M. y SANMARTÍ, N. (1999). Cómo escribir sobre los experimentos. Actas de la 2 a Reunión de la ESERA. Kiel. Alemania.
LEMKE, J. (1997). Aprender a hablar ciencia. Barcelona: Paidós. (Ed. orig. en inglés de 1993)

MONEREO FONT, C. (1995). Enseñar a conciencia. ¿Hacia una didáctica metacognitiva? Aula de Innovación Educativa, 34, pp. 74-80.

PIAGET, J. e INHELDER, B. (1959). La genèse des structures logiques élémentaires. París: Niestlé.

PITTMAN, K.M. (1999). Generated analogies: another way of knowing? Journal of Research in Science Teaching, 36(1), pp. 1-22.

PORLÁN, R. (1998). Pasado, presente y futuro de la didáctica de las ciencias. Enseñanza de las Ciencias, 16(1), pp. 175-185.

SANMARTÍ, N. e IZQUIERDO, M. (1997). Reflexiones en torno a un modelo de ciencia escolar. Investigación en la Escuela, 32, pp. 51-62.

VYGOTSKY, L. (1993). Estudio del desarrollo de los conceptos científicos en la edad infantil. Problemas de psicología general, cap. 6, pp. 248-255, en Obras Escogidas. Madrid: Aprendizaje Visor.

[Artículo recibido en noviembre de 1999 y aceptado en diciembre de 2000.] 


\section{APÉNDICE}

Cuadro I

Material de base para el registro escrito del MDA sobre el modelo representativo de la dinámica celular.

Dada la siguiente lista de agentes externos e internos de una vivienda, describa de qué manera puede entrar o salir cada uno de ellos de la misma. En la descripción tenga en cuenta, además del lugar de entrada o salida, las condiciones y operaciones que deben darse para que cada uno de dichos agentes se desplace hacia o desde el interior de la casa. Vuelque sus conclusiones en el siguiente cuadro:

\begin{tabular}{|l|l|l|}
\hline Agente & Lugarde paso & $\begin{array}{c}\text { iSe desplaza por medios propios? iMediante qué acciones? } \\
\text { iDe qué dep en de que se desplace? iAlguien ayuda? }\end{array}$ \\
\hline Humo & & \\
\hline $\begin{array}{l}\text { Moscas y } \\
\text { mosquitos }\end{array}$ & & \\
\hline Aire & & \\
\hline Piano & & \\
\hline Médico & & \\
\hline Carta & & \\
\hline Ladrón & & \\
\hline Elefante & & \\
\hline $\begin{array}{l}\text { Cobrador de } \\
\text { impuestos }\end{array}$ & & \\
\hline Residuos & & \\
\hline
\end{tabular}


Posible registro escrito resultante de la primera etapa del MDA sobre el modelo representativo de la dinámica celular.

\begin{tabular}{|c|c|c|c|}
\hline \multirow[t]{2}{*}{ Agente } & \multirow[t]{2}{*}{ Lugar de paso } & \multicolumn{2}{|c|}{$\begin{array}{c}\text { ¿Se desplaza por medios propios? ¿Mediante qué acciones? } \\
\text { ¿De qué d ep en de que se desplace? ¿Alguien ayuda? }\end{array}$} \\
\hline & & $\begin{array}{l}\text { Respuestas posibles } \\
\text { de los alumnos }\end{array}$ & $\begin{array}{l}\text { Debería llegarse } \\
\text { al siguiente consen so }\end{array}$ \\
\hline Humo & $\begin{array}{l}\text { Aberturas, } \\
\text { rendijas }\end{array}$ & $\begin{array}{l}\text { Se desplaza por medios propios, entra ayudado } \\
\text { por: } \\
\text { - el viento } \\
\text { - la temperatura } \\
\text { - empujado por el aire }\end{array}$ & $\begin{array}{l}\text { Transporte por difusión, por } \\
\text { gradiente de concentración }\end{array}$ \\
\hline $\begin{array}{l}\text { Moscas y } \\
\text { mosquitos }\end{array}$ & Aberturas & $\begin{array}{l}\text { Se desplazan por medios propios (agitan las alas), } \\
\text { entran ayudados por: } \\
\text { - la temperatura del cuerpo } \\
\text { - el calor } \\
\text { - el olor a sangre }\end{array}$ & $\begin{array}{l}\text { Transporte que requiere } \\
\text { gasto de energía }\end{array}$ \\
\hline Aire & $\begin{array}{l}\text { Aberturas, } \\
\text { rendijas, } \\
\text { poros }\end{array}$ & $\begin{array}{l}\text { Se desplaza por medios propios. Ayudado por: } \\
\text { - el viento } \\
\text { - la temperatura } \\
\text { - el movimiento }\end{array}$ & $\begin{array}{l}\text { Transporte por difusión, } \\
\text { por diferencia de presiones }\end{array}$ \\
\hline Piano & $\begin{array}{l}\text { Aberturas } \\
\text { hechas } \\
\text { o por } \\
\text { modificar }\end{array}$ & $\begin{array}{l}\text { No se desplaza por medios propios. Si entra, } \\
\text { no sale; para entrar debe ser ayudado por: } \\
\text { - personas } \\
\text { - deben utilizarse poleas } u \text { otros aparatos } \\
\text { - puede requerirse hacer aberturas especiales } \\
\text { y luego cerrarlas }\end{array}$ & $\begin{array}{l}\text { Entrada mediante procesos } \\
\text { o artefactos específicos }\end{array}$ \\
\hline Médico & Puerta & $\begin{array}{l}\text { Se desplaza por medios propios; para entrar } \\
\text { debe ser ayudado por: } \\
\text { - un llamado de necesidad } \\
\text { - alguien que abra la puerta }\end{array}$ & $\begin{array}{l}\text { Entrada por necesidad } \\
\text { interna, se le «permite } \\
\text { entrar»= concepto de } \\
\text { selectividad }\end{array}$ \\
\hline Carta & $\begin{array}{l}\text { Puerta } \\
\text { (entra o sale); } \\
\text { también puede } \\
\text { entrar por el } \\
\text { buzón }\end{array}$ & $\begin{array}{l}\text { No se desplaza por medios propios. Para entrar } \\
\text { debe ser ayudado por: } \\
\text { - personas que abren la puerta al cartero } \\
\text { - el buzón. } \\
\text { Para salir también necesita que alguien la lleve }\end{array}$ & $\begin{array}{l}\text { Entrada y salida por métodos } \\
\text { o procesos diferentes }\end{array}$ \\
\hline Ladrón & $\begin{array}{l}\text { Cualquier tipo } \\
\text { de aberturas } \\
\text { existentes o las } \\
\text { que él hace }\end{array}$ & $\begin{array}{l}\text { Se desplaza por medios propios, debe ser } \\
\text { ayudado por: } \\
\text {-ganzúas, trepanadoras, artefactos que simulan } \\
\text { elementos verdaderos (ardides) }\end{array}$ & $\begin{array}{l}\text { Entra engañando sistemas } \\
\text { de defensa y seguridad }\end{array}$ \\
\hline Elefante & $\begin{array}{l}\text { No hay aberturas } \\
\text { previstas }\end{array}$ & $\begin{array}{l}\text { No puede entrar, a menos que destruya una } \\
\text { parte de la casa }\end{array}$ & $\begin{array}{l}\text { Hay cosas que no entran, } \\
\text { la impermeabilidad es } \\
\text { consecuencia de la } \\
\text { selectividad }\end{array}$ \\
\hline $\begin{array}{l}\text { Cobrador } \\
\text { de impuestos }\end{array}$ & Aberturas & $\begin{array}{l}\text { Se desplaza por medios propios, llega por: } \\
\text { - una necesidad externa a la casa } \\
\text { - necesita que alguien le abra la puerta }\end{array}$ & $\begin{array}{l}\text { Entrada por necesidad } \\
\text { externa, otra vez el concepto } \\
\text { de selectividad }\end{array}$ \\
\hline Residuos & Aberturas & $\begin{array}{l}\text { Algunos tipos se desplazan por: } \\
\text { - medios propios (polvo) y otros no (basura) } \\
\text { - alguien tiene que juntarlos antes } \\
\text { de sacarlos }\end{array}$ & $\begin{array}{l}\text { Transporte activo, } \\
\text { para sacarlos hay } \\
\text { que agruparlos }\end{array}$ \\
\hline
\end{tabular}

